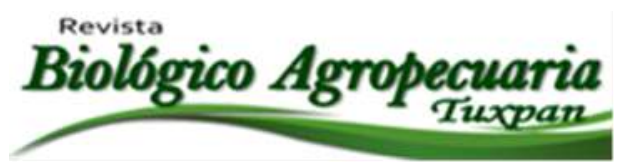

\title{
Conservación de los recursos fitogenéticos: uso inteligente de la biodiversidad
}

Conservation of phytogenetic resources: smart use of biodiversity

Octavio-Aguilar Pablo ${ }^{1 凶}$, García-Montes Mario Adolfo ${ }^{1}$, Galván-Hernández Dulce María ${ }^{1}$, Macedo-Villarreal

Manuel Alejandroํㅗㅇ, Sánchez-González Arturo ${ }^{1}$

${ }^{1}$ Universidad Autónoma del Estado de Hidalgo, Centro de Investigaciones Biológicas, Carretera PachucaTulancingo Km. 4.5 Ciudad del Conocimiento, Col. Carboneras, Mineral de la Reforma, Hidalgo, México C.P. 42184.

凶Autor de correspondencia: pablo_aguilar9900@uaeh.edu.mx

Recibido: 13/07/2018

Aceptado: 12/09/2018

\section{RESUMEN}

Los recursos fitogenéticos están representados por toda la variedad y combinaciones posibles de rasgos que permiten a las especies vegetales adaptarse a los cambios en el medio; a mayor cantidad de rasgos y combinaciones, mayor es la probabilidad de que una especie pueda subsistir. El estado de Hidalgo presenta condiciones topográficas que lo hacen mega-diverso, ya que crean un mosaico de paisajes que someten a las plantas a diferentes presiones de selección. Sin embargo, de toda esa diversidad muy poca ha sido evaluada a nivel genético, por lo que en este trabajo se presentará un resumen de tres estudios específicos que se han realizado en la región hidalguense. En general, el uso inadecuado de los recursos (Cedrela odorata), el aislamiento (Fagus grandifolia subsp. mexicana) y la perturbación (Ceratozamia fuscoviridis) son los principales problemas a los que se enfrentan los recursos fitogenéticos del Estado de Hidalgo.

Palabras clave: Poblaciones, recursos genéticos, plantas, Hidalgo.

\begin{abstract}
The phytogenetic resources are being represented by all possible variation and combinations of traits that are permitted to the vegetal species adapt it to environment changes, to highest quantity of traits and combinations, highest probabilities had the plants to subsist. Hidalgo State's topographical conditions it's make mega-diverse since they create a mosaic of landscapes that subject plant species to different selection pressures. However, of all diversity, so few have been evaluated at genetic level, this work summarices some specific studies that have been conducted in Hidalgo region. In general, inadequate use of the resources (Cedrela odorata), isolation (Fagus grandifolia subsp. mexicana), and disturbance (Ceratozamia fuscoviridis) are the main problems on the phytogenetic resources of Hidalgo State.
\end{abstract}

Keywords: Populations, genetic resources, plants, Hidalgo. 


\section{INTRODUCCIÓN}

Todos los individuos tienen rasgos característicos que los distinguen, por ejemplo es posible encontrar en un cultivo organismos con mayor producción, con mejor calidad en sus frutos o que son capaces de sobrevivir en condiciones de sequía; esas capacidades son codificadas por el DNA, por lo que es importante identificar esas variantes para seleccionarlas en un contexto productivo y darles un uso adecuado (Emperaire y Peroni, 2007). La identificación de la variabilidad genética de interés agroforestal requiere de herramientas moleculares capaces de mostrar pequeñas diferencias entre los individuos que permitan calcular que tan frecuentemente encontramos genes deseables dentro de una población. De esta manera, es posible contrastar dichas frecuencias para identificar fuentes de variación genética con uso potencial, evaluar regiones geográficas amenazadas por la pérdida de variabilidad e identificar problemas puntuales a mediano y largo plazo asociados con el manejo, la degradación ambiental o las actividades antropogénicas (Hughes et al., 2008).

Cuantificar la variación genética es una tarea difícil pues depende del esfuerzo de muestreo al interior de las poblaciones, la precisión de la herramienta molecular utilizada, así como de la extensión del área y el número de poblaciones a comparar; cualquier deficiencia en estos niveles tiende a subrepresentar la diversidad y a sobreestimar el grado de amenaza de los recursos, por lo que una prospección adecuada debe ser el primer paso antes de iniciar un estudio genético poblacional (Octavio-Aguilar et al., 2017a). Una vez considerados los puntos anteriores, la diversidad puede ser medida usando diferentes indicadores, entre los que destacan: la heterocigosis observada y esperada (frecuencia de individuos con alelos diferentes heredados de padre y madre), el índice de diversidad (medida de las diferencias entre individuos) y el coeficiente de fijación (que tanta variación se distribuye dentro de la población y que proporción proviene de fuentes externas) (Hartl y Clark, 1997). Con estos indicadores es posible valorar cuales poblaciones son más o menos diversas y por lo tanto susceptibles o no de ser aprovechadas.

Cuando dos o más poblaciones son similares en cuanto a su nivel de variación, se debe considerar que esto no significa que sean idénticas. Por ejemplo, si dos poblaciones tienen 85 alelos solo algunos estarán compartidos, lo que permite establecer una medida de similitud. Al respecto, existe un modelo que predice la proporción de alelos compartidos como una función directa de la distancia geográfica, lo que se conoce como aislamiento por distancia. Bajo esta premisa, la diversidad genética se distribuye como un mosaico donde las barreras, la proximidad, la capacidad de radiación y las condiciones ambientales particulares de cada sitio pueden incrementar o diluir la similitud genética (Hartl y Clark, 1997). Entonces, tanto el nivel de variación como la distribución de la misma en el espacio geográfico; resultan ser aspectos exclusivos y necesarios para entender los procesos que hacen a una planta susceptible de conservación, uso y/o manejo. Por ello, en este trabajo se presentan tres estudios de caso particulares a la región hidalguense, donde se ejemplifican procesos ecológicos y evolutivos que permiten dilucidar el potencial de 
aprovechamiento sustentable e inteligente de los recursos fitogenéticos.

\section{MATERIALES Y MÉTODOS}

La colecta del material biológico se realizó en varios puntos del estado de Hidalgo y Veracruz considerando condiciones contrastantes para poder evaluar el efecto de las actividades antropogénicas sobre los recursos fitogenéticos (Tabla 1).

Tabla 1. Sitios de colecta para las especies analizadas. Se especifican las actividades antropogénicas que afectan cada zona y el aislamiento en el caso de Fagus grandifolia subsp. mexicana.

\begin{tabular}{|c|c|c|c|c|}
\hline Sitio & Actividad antropogénica & Latitud & Longitud & Altitud \\
\hline \multicolumn{5}{|c|}{ Cedrela odorata } \\
\hline Huextetitla & $\begin{array}{l}\text { Cultivo con fuentes semilleras ajenas al } \\
\text { estado de Hidalgo. }\end{array}$ & $21^{\circ} 2^{\prime} 27.78^{\prime \prime}$ & $98^{\circ} 20^{\prime} 56.22^{\prime \prime}$ & 493 \\
\hline San Pedro & $\begin{array}{l}\text { Cultivo con fuentes semilleras propias } \\
\text { de la región. }\end{array}$ & $21^{\circ} 9^{\prime} 58.57^{\prime \prime}$ & $98^{\circ} 34^{\prime} 58.87^{\prime \prime}$ & 737 \\
\hline Chiconcoac & $\begin{array}{l}\text { Población natural sin registro de } \\
\text { aprovechamiento. }\end{array}$ & $21^{\circ} 0 ' 2275^{\prime \prime}$ & $98^{\circ} 47^{\prime} 54.67^{\prime \prime}$ & 327 \\
\hline Coatenahuatl & $\begin{array}{l}\text { Población natural sin registro de } \\
\text { aprovechamiento. }\end{array}$ & $21^{\circ} 0^{\prime} 10.76^{\prime \prime}$ & $98^{\circ} 47^{\prime} 58.99^{\prime \prime}$ & 314 \\
\hline Coyolapa & $\begin{array}{l}\text { Población natural con aprovechamiento } \\
\text { por más de } 10 \text { años. }\end{array}$ & $20^{\circ} 56^{\prime} 57.8^{\prime \prime}$ & $98^{\circ} 31^{\prime} 44.97^{\prime \prime}$ & 401 \\
\hline Tamala & $\begin{array}{l}\text { Población natural con aprovechamiento } \\
\text { por más de } 25 \text { años }\end{array}$ & $21^{\circ} 4^{\prime} 14.41^{\prime \prime}$ & $98^{\circ} 17^{\prime} 12.04^{\prime \prime}$ & 328 \\
\hline \multicolumn{5}{|c|}{ Ceratozamia fuscoviridis 1712} \\
\hline Demañi & Población conservada & $20^{\circ} 55^{\prime} 26.83^{\prime \prime}$ & $98^{\circ} 59^{\prime} 38.88^{\prime \prime}$ & 1712 \\
\hline $\begin{array}{l}\text { Palo Perdido } \\
1\end{array}$ & Pastoreo extensivo & $20^{\circ} 56^{\prime} 25.9^{\prime \prime}$ & $99^{\circ} 0^{\prime} 11.38^{\prime \prime}$ & 1920 \\
\hline $\begin{array}{l}\text { Palo Perdido } \\
2\end{array}$ & Población conservada & $20^{\circ} 56^{\prime} 35.74^{\prime \prime}$ & $99^{\circ} 0 ' 17.73^{\prime \prime}$ & 1840 \\
\hline El Duraznito & Extracción intensiva de madera & $20^{\circ} 56^{\prime} 15.41^{\prime \prime}$ & $99^{\circ} 0^{\prime} 40.42^{\prime \prime}$ & 1480 \\
\hline \multicolumn{5}{|c|}{ Fagus grandifolia subsp. mexicana } \\
\hline La Mojonera & $\begin{array}{l}\text { Municipio de Zacualtipán de Ángeles, } \\
\text { Hidalgo }\end{array}$ & $20^{\circ} 38^{\prime} 0.33^{\prime \prime}$ & $99^{\circ} 36^{\prime} 51.8^{\prime \prime}$ & 1780 \\
\hline Medio Monte & $\begin{array}{l}\text { Municipio de San Bartolo Tutotepec, } \\
\text { Hidalgo }\end{array}$ & $20^{\circ} 24^{\prime} 50^{\prime \prime}$ & $98^{\circ} 14^{\prime} 24^{\prime \prime}$ & 1800 \\
\hline El Gosco & $\begin{array}{l}\text { Municipio de Tenango de Doria, } \\
\text { Hidalgo }\end{array}$ & $20^{\circ} 19^{\prime} 37.8^{\prime \prime}$ & $98^{\circ} 14^{\prime} 57.1^{\prime \prime}$ & 1557 \\
\hline $\begin{array}{l}\text { Volcán de } \\
\text { Acatlán }\end{array}$ & $\begin{array}{l}\text { Municipio de Acatlán, Veracruz } \\
\text { (aislada) }\end{array}$ & $19^{\circ} 40^{\prime} 46.9^{\prime \prime}$ & $96^{\circ} 51^{\prime} 9.8^{\prime \prime}$ & 1840 \\
\hline
\end{tabular}

La extracción del material genético se realizó con el método de Doyle y Doyle (1987), modificado para cada especie (SánchezHernández y Gaytán-Oyarzín, 2006; SánchezCoello et al., 2012). La variación genética fue obtenida mediante amplificación con microsatélites específicos para cada especie: Cedrela odorata nueve microsatélites (Hernández et al., 2008); Fagus grandifolia subsp. mexicana seis microsatélites (Pastorelli 
et al., 2003; Li-Ping et al., 2012); Ceratozamia fuscoviridis nueve microsatélites (Meerow y Nakamura, 2007). Los datos se analizaron con el programa GenAlEx (Pekall y Smouse, 2012) para obtener los indicadores de diversidad y estructura.

\section{RESULTADOS}

\section{Cedro rojo (Cedrela odorata, Meliaceae)}

Este árbol, nativo de América tropical, es apreciado por la durabilidad, color y aroma de su madera. Se encuentra listado en el apéndice III del Convenio sobre el Comercio Internacional de Especies Amenazadas de Fauna y Flora Silvestres (CITES, 2016), por lo que existe un comercio activo aunque limitado en algunos países, incluido México (NegrerosCastillo et al., 2010). Para cubrir la demanda sin afectar a las poblaciones nativas, se han implementado cultivos como una opción sustentable de manejo. En el presente trabajo, se contrastó la variabilidad y estructura genética de poblaciones naturales, poblaciones que han sido sometidas a extracción y cultivos; para determinar el efecto que el manejo ha tenido sobre el acervo genético de la especie.

Los resultados muestran que las poblaciones naturales mantienen una mayor heterocigosis observada $(\mathrm{HO}=0.88 \pm 0.07)$, presentan exogamia, es decir; sus fuentes de variación son externas a la población $(\mathrm{F}=-0.1$ \pm 0.11 ), y su coeficiente de diferenciación es bajo (FST $=0.046$ ), por lo que comparten variantes alélicas propias de la región. En contraste, las poblaciones que han sido sometidas a extracción, han sufrido una pérdida de heterocigosis con respecto a las poblaciones naturales $(\mathrm{HO}=0.84 \pm 0.03)$ y presentan endogamia $(F=0.02 \pm 0.06)$, aunque siguen conservando cierta similitud genética con respecto a las poblaciones naturales $(\mathrm{FST}=$ 0.043 ) correlacionada con la distancia.

En cuanto a los cultivos existe un contraste importante dependiendo de la fuente; si el cultivo proviene de la región, la variabilidad genética es alta $(\mathrm{HO}=0.89 \pm 0.02)$ $\mathrm{y}$ las diferencias se diluyen $(\mathrm{FST}=0.037)$, pero si proviene de una fuente externa la variación es menor $(\mathrm{HO}=0.87 \pm 0.03)$, se incrementa la exogamia $(F=-0.07 \pm 0.05)$ y las diferencias con respecto a las poblaciones nativas (FST $=$ 0.059) (Tabla 2).

Entonces, el efecto principal de iniciar cultivos con semillas externas es la pérdida de variabilidad genética, lo que incrementa su diferenciación con respecto al resto de las poblaciones, haciéndolas susceptibles al ambiente y de baja calidad. Pero, si el cultivo proviene de fuentes semilleras múltiples y de origen autóctono, tienen mayor diversidad. Esta estrategia resulta mejor para la conservación de la diversidad genética, siempre que se tenga un ingreso constante de individuos procedentes de la fuente original para no perder los niveles de variación (Flores et al., 2007). En conclusión, para Cedrela odorata, el uso inadecuado de los recursos compromete su variabilidad fitogenética en el corto plazo e incrementa la diferenciación al cultivar semillas externas a la región. 
Tabla 2. Comparación de la variabilidad genética entre poblaciones naturales, poblaciones sometidas a extracción y cultivos de cedro rojo ( $C$. odorata) en el estado de Hidalgo. N: número de individuos evaluados, Na: promedio de alelos por locus, Ne: número efectivo de alelos por locus, I: índice de similitud, HO: heterocigosis observada, HE: heterocigosis esperada, F: índice de fijación (endogámia), EHW: equilibrio de Hardy-Weimberg.

\begin{tabular}{ccccccccc}
\hline Condición & $\mathbf{N}$ & $\mathbf{N a}$ & $\mathbf{N e}$ & $\mathbf{I}$ & HO & HE & $\mathbf{F}$ & EHW \\
\hline Naturales & 17 & 8 & 5.37 & 1.82 & 0.88 & 0.81 & -0.1 & $\mathrm{~ns}$ \\
Extracción & 22 & 9 & 5.89 & 1.91 & 0.82 & 0.84 & 0.02 & $* * *$ \\
Cultivos & 24 & 9 & 6.11 & 1.92 & 0.88 & 0.83 & -0.07 & $*$ \\
\hline \multicolumn{7}{c}{$n s:$ significativo; $* * *$} & $<0.001$ & de acuerdo con la prueba de Chi cuadrada.
\end{tabular}

Haya (Fagus grandifolia subsp. mexicana, Fagaceae)

Las poblaciones de este árbol con afinidad boreal se distribuyen de forma discontinua desde Canadá hasta México, con divergencia estimada de más de dos millones de años (Plioceno). En el país se han registrado solo siete localidades restringidas a la Sierra Madre Oriental en los estados de Nuevo León, Tamaulipas, Hidalgo y Veracruz (Ortiz-Quijano et al., 2016). Las localidades mexicanas se encuentran aisladas y fragmentadas, por lo que en este trabajo se evaluó el efecto del aislamiento sobre la diversidad genética de cuatro poblaciones al sur de la distribución.

Los resultados muestran que las poblaciones hidalguenses tienen mayor diversidad genética $(\mathrm{HO}=0.66 \pm 0.075)$, lo que coincide con la cantidad de individuos contabilizados y la extensión de los manchones de bosque de haya (Ortiz-Quijano et al., 2016). Por el contrario, la población más aislada, fragmentada y restringida en cuanto a cantidad de individuos, localizada en Acatlán, Veracruz; posee menor diversidad $(\mathrm{HO}=0.56 \pm 0.145)$ y mayor endogamia $(\mathrm{F}=0.226)$; sin embargo, no se observan diferencias significativas con respecto a las poblaciones de Hidalgo (FST = 0.053), lo que sugiere un origen común. Destaca el hecho de que las poblaciones en los extremos de la distribución presentan mayor endogamia (al norte La Mojonera, Hidalgo y al sur Acatlán, Veracruz) y ninguna de las poblaciones analizadas se encuentra en equilibrio de Hardy-Weimberg, lo que implica que la especie es susceptible al aislamiento, particularmente en las zonas limítrofes de su distribución (Tabla 3).

Estos resultados destacan la importancia de las poblaciones de haya hidalguenses como un reservorio de variabilidad genética, información importante para cualquier programa de manejo y conservación. En conclusión, la principal amenaza sobre el acervo genético de Fagus grandifolia supsp. mexicana, es el aislamiento y la fragmentación, particularmente en los extremos de la distribución. 
Tabla 3. Comparación de la variabilidad genética entre poblaciones aisladas de haya (F. grandifolia subsp mexicana). N: número de individuos evaluados, Na: promedio de alelos por locus, Ne: número efectivo de alelos por locus, I: índice de similitud, HO: heterocigosis observada, HE: heterocigosis esperada, F: índice de fijación (endogamia), EHW: equilibrio de Hardy-Weimberg.

\begin{tabular}{ccccccccc}
\hline Localidad & N & Na & Ne & I & HO & HE & F & EHW \\
\hline La Mojonera, Hgo. & 23 & 6.66 & 4.37 & 1.54 & 0.59 & 0.73 & 0.22 & $* * *$ \\
Medio Monte, Hgo. & 30 & 8.83 & 5.04 & 1.75 & 0.64 & 0.76 & 0.17 & $* * *$ \\
El Gosco, Hgo. & 30 & 7.5 & 4.34 & 1.63 & 0.74 & 0.74 & 0.01 & $* * *$ \\
Acatlán, Ver. & 38 & 8 & 4.08 & 1.53 & 0.56 & 0.7 & 0.22 & $* * *$
\end{tabular}

ns: no significativo no hay ningún valor ns en la tabla; $* * * p<0.001$ de acuerdo con la prueba de Chi cuadrada.

\section{Teosintle (Ceratozamia fuscoviridis)}

Esta cícada ha sido usada frecuentemente con fines ornamentales y en época de escases ocasionalmente ha brindado sustento alimenticio a los pobladores como sustituto del maíz (Vite et al., 2013). Sin embargo, como la mayoría de las cícadas del género, requieren ambientes húmedos y con amplia cobertura vegetal para que sus semillas puedan germinar, pues tienen un periodo de latencia limitado por la humedad (Sánchez-Tinoco et al., 2000). Esto hace que las especies del género sean susceptibles a cambios antropogénicos (RiveraFernández et al., 2012), por lo que en este trabajo se evaluó el efecto de la perturbación sobre el acervo genético de la planta.

Se evaluaron cuatro poblaciones con diferente grado de perturbación, determinado mediante un indicador métrico propuesto por Martorell y Peters (2005). La población con el mayor grado de perturbación conserva niveles aceptables de variabilidad genética $(\mathrm{HO}=0.55$ \pm 0.29) con respecto a la población más conservada $(\mathrm{HO}=0.41 \pm 0.21)$. Sin embargo, la población más aislada geográficamente mostró el nivel más bajo de variabilidad $(\mathrm{HO}=0.24 \pm$
$0.21)$ y la mayor endogamia $(F=0.7 \pm 0.25)$. Además, las poblaciones geográficamente más cercanas mantienen una identidad genética elevada (FST $=0.096)$, lo que parece amortiguar el efecto de la perturbación (Tabla 4). Estos resultados mostraron que para una especie de vida larga, más de 60 años para alcanzar la etapa reproductiva, la perturbación causa menos impacto que para especies de crecimiento rápido. No obstante, en un estudio previo con otros marcadores más extensos en el área del DNA evaluado (datos no mostrados), si se muestra una correlación entre la pérdida de diversidad y el grado de perturbación (IslasBarrios, 2016) aunque estos marcadores no permiten calcular la endogámia ni la diferenciación, por lo que se requiere de un estudio complementario como el mostrado en este trabajo. En ambos casos, ninguna población se encuentra en equilibrio, por lo que la especie es susceptible de conservación. En conclusión, la principal amenaza sobre la diversidad genética de Ceratozamia fuscoviridis, es la perturbación pero con un efecto amortiguado por la longevidad de la planta. 
Tabla 4. Comparación de la variabilidad genética entre poblaciones con diferente grado de perturbación de teosintle (C. fuscoviridis). N: número de individuos evaluados, Na: promedio de alelos por locus, Ne: número efectivo de alelos por locus, I: índice de similitud, HO: heterocigosis observada, HE: heterocigosis esperada, F: índice de fijación (endogámia), EHW: equilibrio de Hardy-Weimberg.

\begin{tabular}{ccccccccc}
\hline $\begin{array}{c}\text { Grado de perturbación } \\
\text { (escala 0-14) }\end{array}$ & $\mathbf{N}$ & $\mathbf{N a}$ & $\mathbf{N e}$ & $\mathbf{I}$ & $\mathbf{H O}$ & $\mathbf{H E}$ & $\mathbf{F}$ & $\mathbf{E H W}$ \\
\hline 2.32 & 29 & 6.8 & 4.85 & 1.7 & 0.41 & 0.79 & 0.47 & $* * *$ \\
3.9 & 29 & 6.2 & 3.57 & 1.41 & 0.24 & 0.68 & 0.7 & $* * *$ \\
5.37 & 23 & 4.8 & 3.77 & 1.39 & 0.31 & 0.73 & 0.58 & $* * *$ \\
7.37 & 24 & 7.4 & 4.48 & 1.61 & 0.55 & 0.73 & 0.24 & $* * *$ \\
ns: no significativo; & $* * * p$ & 0.001 de acuerdo con la prueba de Chi cuadrada. & \multicolumn{7}{c}{}
\end{tabular}

\section{DISCUSIÓN}

Durante el I Simposio en Conservación y Uso de los Recursos Naturales, Mineral de la Reforma, Hidalgo 2018; este trabajo fue presentado como conferencia magistral, mostrando además algunos datos interesantes sobre la manera en que la diversidad genética se distribuye en el paisaje asociada a procesos de aislamiento, pero también a la acción del hombre en su afán por conservar.

Los recursos fitogenéticos incluyen prácticamente a cualquier recurso vegetal en una región, el uso de los mismos dependerá en principio del conocimiento ancestral y de la experiencia productiva, pero para realmente utilizar de manera inteligente dichos recursos, se debe garantizar que las futuras generaciones tengan acceso a los beneficios. Como muestran los resultados, la base genética de las poblaciones es afectada por varias actividades antropogénicas, incluso algunas encaminadas a la conservación, como en el caso de Cedrela odorata; cualquier modificación de la conectividad resulta en una potenciación de los procesos intrínsecos a las poblaciones (endogámia, deriva, etc.) generando un riesgo asociado a esos procesos que se incrementa en cada generación.

Los resultados presentados son solo una pequeña selección de varios trabajos realizados a la fecha en el Laboratorio de Genética de la UAEH y además, solo se muestran los datos más relevantes que permiten identificar procesos generales asociados al manejo y la degradación ambiental. Como contraste en el suscrito laboratorio, se han evaluado procesos de aislamiento geográfico y pérdida de conectividad por ausencia de polinizadores en dos especies vegetales: Zamia inermis y Dichromanthus aurantiacus, corroborando que el efecto del aislamiento resulta en un incremento en el riesgo de extinción local. Mediante análisis genéticos ha sido posible identificar elementos divergentes que sugieren especies crípticas (morfológicamente similares pero genéticamente distintas) en el complejo Magnolia dealbata, procesos de especiación también asociados al aislamiento genético. Además, se han identificado procesos de selección direccional y balanceadora que responden al cambio climático en Ceratozamia kuesteriana y $C$. zaragozae, particularmente en las zonas limítrofes de la distribución de las especies, por lo que el efecto de la selección direccional se vería potenciado también por el aislamiento. Los estudios genéticos también han permitido identificar procesos de hibridación entre $C$. furcoviridis y $C$. latifolia que coinciden en una delgada franja de bosque conservado del estado de Hidalgo. 
Contrariamente a los casos mostrados, este proceso de hibridación estaría diluyendo la divergencia e incrementando la variabilidad de las especies por lo que la conectividad resultaría en un método de resguardo y protección de los recursos genéticos.

La genética de poblaciones permitió evaluar el efecto de la fragmentación, cambio climático y perturbación en el helecho Dicksonia sellowiana, llegando nuevamente a la conclusión que la fragmentación por actividades antropogénicas son la principal amenaza sobre los recursos. Este listado de trabajos, enfatizan la versatilidad de los estudios genéticos en el contexto de la conservación y el manejo. En todos los casos, las actividades antropogénicas generan una pérdida de la conectividad y procesos de diferenciación con una consecuente pérdida de diversidad genética.

Por otro lado, cada aproximación al estudio de la variabilidad debe usar sus propias herramientas para identificar los procesos particulares que condicionen o permitan el uso de los recursos fitogenéticos. Por ejemplo, la variación espacial a escala fina requiere herramientas de correlación métrica-genética cuyo objetivo es identificar el área de dispersión, vecindarios familiares, pérdida de conectividad y patrones de distribución espacial (Octavio-Aguilar et al., 2017b). La reducción del tamaño poblacional por eventos recientes, requiere del uso de modelos bayesianos que permitan identificar la probabilidad de que un gen tenga más variación de la que se esperaría por eventos aleatorios (Cornuet y Luikart, 1996). La similitud entre poblaciones hoy en día, se basa en análisis de asignación probabilística de los individuos (Pritchard et al., 2000) y en medidas de similitud multimétricas (Jombart et al., 2010). La evaluación de la divergencia entre poblaciones causada por selección natural, requiere la identificación de alelos exclusivos sobre-representados en localidades con condiciones ambientales extremas, alejadas de lo esperado en un modelo de neutralidad (Foll y Gaggiotti, 2008). Por todo lo anterior, se espera en un futuro complementar las evaluaciones mostradas, considerando más análisis para generar publicaciones específicas. Sin embargo, la información generada requiere de programas para la implementación y uso racional de los recursos, por lo que la transferencia del conocimiento aún se encuentra desvinculada del componente social y productivo.

\section{CONCLUSIONES}

El uso inteligente de los recursos fitogenéticos requiere establecer claramente el efecto que las actividades antropogénicas tienen sobre la variación genética. En general, la pérdida de continuidad ya sea por extracción y uso (cedrela odorata), por fragmentación y aislamiento (Fagus grandifolia subsp. mexicana) o por degradación ambiental y perturbación (Ceratozamia fuscoviridis); incrementan la probabilidad de extinción local y resultan ser la principal amenaza sobre los recursos fitogenéticos.

\section{AGRADECIMIENTOS}

Al Consejo Nacional de Ciencia y Tecnología por las becas brindadas a DMGH y MAMV, por el apoyo INFR-252807 otorgado al Cuerpo Académico de Ecología de la UAEH y por el proyecto CB-2011/169141 otorgado a ASG. 


\section{LITERATURA CITADA}

CITES. 2016. Convenio sobre el Comercio Internacional de Especies Amenazadas de Fauna y Flora. Apéndice III. Cedrela odorata. En: https://cites.org/esp/node/29983. Última consulta enero de 2018.

Cornuet, J. M. y Luikart, G. 1996. Description and power analysis of two tests for detecting recent population bottlenecks from allele frequency data. Genetics 144: 2001-2014. https://doi.org/10.1093/genetics/144.4.2001

Doyle, J. J. y Doyle, J. L. 1987. A rapid DNA isolation procedure for small quantities of fresh leaf tissue. Phytochemistry Bulletin 19: 11-15.

Emperaire, L. y Peroni, N. 2007. Traditional management of agrobiodiversity in Brazil: A case study of manioc. Human Ecology 35(6): 761-768. https://doi.org/10.1007/s10745-007-9121$\mathrm{X}$

Flores, G. J. G., Moreno, G. D. A. y Quiñones, CH. A. 2003. Selección de criterios para evaluar el manejo forestal sustentable. Jalisco, México. Publicación Especial No.

2. Centro de Investigación Regional del Pacifico Centro. INIFAP 46 pp.

Foll, M. y Gaggiotti, O. 2008. A genome-scan method to identify selected loci appropriate for both dominant and codominant markers: a Bayesian perspective. Genetics 180: 977-993. https://doi.org/10.1534/genetics.108.0922 21

Hartl, D. L. y Clark, A. G. 1997. Principles of population genetics, 4th edition. Sinauer Associates Press, Sunderland MA, USA, $652 \mathrm{pp}$.
Hernández, G., Buonamici, A., Wlaker, K., Vendramin, G. G., Navarro, C. y Carvers, S. 2008. Isolation and characterization of microsatellite markers for Cedrela odorata L. (Meliaceae), a high value neotropical tree. Conservation Genetics 9: 457-459. https://doi.org/10.1007/s10592-007-9334-y

Hughes, A. R., Inouye, B. D., Johnson, M. T. J., Underwood, N. y Vellend, M. 2008. Ecological consequences of genetic diversity. Ecology Letters 11(6):

609-623.

https://doi.org/10.1111/j.1461-0248.20 08.01179.X

Islas-Barrios, Y. 2016. Efecto de la perturbación sobre la variabilidad y estructura genética de Ceratozamia fuscoviridis Moore (Cycadales, Zamiaceae): especie endémica y amenazada del estado de Hidalgo. Tesis de Maestría, Postgrado en Biodiversidad y Conservación, Área Académica de Biología, Universidad Autónoma del Estado de Hidalgo

Jombart, T., Devillard, S. y Balloux, F. 2010. Discriminant analysis of principal components: a new method for the analysis of genetically structured populations. BMC Genetic 11: 94. https://doi.org/10.1186/1471-2156-11-94

Li-Ping, J., Huei-Chuan, S. y Yu-Chung, C. 2012. Microsatellite primers for the endangered beech tree Fagus hayatae (fagaceae). American Journal of Botany e453-e456. https://doi.org/10.3732/ajb.1200118

Martorell, C. y Peters, E. M. 2005. The measurement of chronic disturbance and its effect on the threatened cactus Mammillaria pectinifera. Biological Conservation 124: 199-207. https://doi.org/10.1016/j.biocon.2005.01.0 25 
Negreros-Castillo, P., Apodaca-Martínez, M. y Mize, C. W. 2010. Effect of substrate and density on the quality of seedlings of Mexican cedar, mahogany and roble (Tabebuia). Maderas yBosques 16: 7-18.https://doi.org/10.21829/yb.2010.1 621169

Octavio-Aguilar, P., Iglesias-Andreu, L. G., Vovides, A. P. y Rivera-Fernández, Y. A. 2017a. Zamia inermis, the more threatened cycad of Mexico. Cuadernos de Biodiversidad 52: 1-5.

Octavio-Aguilar, P. Iglesias-Andreu, L. G., Núñez, de C-G. F. F. y GalvánHernández, D. M. 2017b. Fine-scale genetic structure of Zamia furfuracea: variation with life-cycle stages. International Journal of Plant Sciences 178(1): 57-66.

https://doi.org/10.1086/689200

Ortiz-Quizano, A. B., Sánchez-González, A., López-Mata, L. y Villanueva-Díaz, J. 2016. Population structure of Fagus grandifolia subsp. mexicana un the cloud forest of Hidalgo State, Mexico. Botanical Sciences 94(3): 483-497. https://doi.org/10.17129/botsci.515

Pastorelli, R., Smulders, M. J. M., Van't Westende, W. P. C., Vosman, B., Giannini, R., Vettori, C. y Vendatmin, G. G. 2003. Characterization of microsatellite markers in Fagus sylvatica L. and Fagus orietalis Lipski. Molecular Ecology Resources 3: 76-78. https://doi.org/10.1046/j.1471-8286.2003. 00355.x

Pekall, R., y Smouse, P. E. 2012. GenAlEx 6.5: genetic analysis in excel. Population Genetics Software for teaching and research. Molecular Ecology Notes 6: 288-295.

https://doi.org/10.1111/j.1471-8286.2005. 01155.x
Pritchard, J. K., Stephens, M. y Donnelly, P. 2000. Inference of population structure using multilocus genotype data. Genetics 155: 945-959.

Rivera-Fernández, A., Octavio-Aguilar, P., Sánchez-Coello, N. G., SánchezVelásquez, L. R., Vázquez-Torres, S. M. e Iglesias-Andreu, L. G. 2012. Estructura poblacional y distribución espacial de Ceratozamia mexicana Brongn. (Zamiaceae) en un ambiente conservado y en uno perturbado. Tropical and Subtropical Agroecosystems 15(2): S110S117.

Sánchez-Coello, N. G., Luna-Rodríguez, M., Vázquez-Torres, M., Sánchez-Velásquez, L. R., Santana-Buzzy, N., OctavioAguilar, P. e Iglesias-Andreu, L. G. 2012. Optimización de un protocolo de aislamiento del DNA y de un sistema de amplificación ISSR-PCR para Ceratozamia mexicana Brongn. (Zamiaceae). Revista Chapingo Serie Ciencias Forestales y del Ambiente 18:123-133.

https://doi.org/10.5154/r.rchscfa.2011.03.0 24

Sánchez-Hernández, C. y Gaytán-Oyarzún, J. C. 2006. Two mini-preparation protocols to DNA extraction from plants with hogh polysaccharide and secondary metabolites. African Journal of Biotechnology 5(20): 18641867.Sánchez-Tinoco, M. Y., Engleman, E. M. y Vovides, A. P. 2000. Cronología reproductora de Ceratozamia mexicana (Cycadales). Boletín de la Sociedad Botánica de México 66: 15-23.

Vite, A., Pulido, M. T. y Flores-Vázquez, J. C. 2013. Estrategia estatal de conservación de las cícadas (Zamiaceae): una propuesta para el estado de Hidalgo, México. Revista de Biología Tropical 61: 11191131. 
Copyright (c) 2018 Pablo Octavio Aguilar, Mario Adolfo G arcia Montes, Dulce Maria Galván Hernández, Maruel Alejandro Macedo Villareal y Arturo Sánchez G onzález

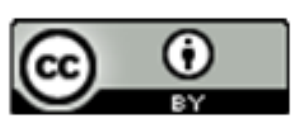

E ste tex to está protegido por una licencia CreativeCommons 4.0

Usted es libre para Compartir —copiar y redistribuir el $\mathrm{m}$ aterial en cualquier medio o formato- y Adaptar el documento —remezclar, transformar y crear a partir del material- para cualquier propósito, incluso para fines com erciales, siempre que cumpla la condición de:

Atribución: Usted debe dar crédito a la obra original de manera adecuada, proporcionar un enlace a la licencia, e in dicar si se han realizado cambios. Puede hacerlo en cualquier forma razonable, pero no de forma tal que sugiera que tiene el apoyo del licenciante olo recibe por el uso que hace de la obra.

Resumenclelicencia - Textocompletodelalicencia 